\author{
Elena I. Nikolaeva \\ Herzen State Pedagogical University \\ Saint-Petersburg, Pussia
}

\title{
Genetics and psychophysiology of ADHD and autism
}

Abstract. The paper discusses the brain mechanisms of autism and attention deficit hyperactivity disorder. It is shown that these disorders are associated with different genetic causes that create certain psychophysiological mechanisms. Nevertheless, their diagnosis is interrelated. Moreover, a child is often first diagnosed with ADHD, and then the diagnosis is changed to "autism spectrum disease".

Among the most common causes of the disease is the behavior of retrotransposons. Retrotransposons (also called transposons via intermediate RNA) are genetic elements that can amplify themselves in the genome. These DNA sequences use a "copy and paste" mechanism, whereby they are first transcribed into RNA and then converted back to identical DNA sequences via reverse transcription, and these sequences are then inserted into the genome at target sites. In humans, retro elements take up $42 \%$ of the DNA.

The conclusion is made that for the formation of an individual profile of gene expression in the neuron, the most important is the phenomenon of somatic mosaicism, due to the process of L1 retrotransposition, in addition to the classical described mechanisms of differentiation. The number of such events and their localization is significant as they are likely to contribute to the development of both autism and ADHD.

Keywords: autism, attention deficit hyperactivity disorder, retrotransposons, reverse transcription

The two diseases -autism and attention deficit hyperactivity disorder (ADHD) - are related to amazing fact, namely, the significant increase in the number of cases was connected to the discovery of penicillin by $\mathrm{A}$. Fleming in the $20^{\text {th }}$ century. Penicillin irreversibly changed the structure of morbidity and mortality in children. In the late $19^{\text {th }}$ century in Russia up to $85 \%$ of infants died in summer from intestinal infections, in the $20^{\text {th }}$ century the mortality rate in civilized countries rarely exceeded 
5-7 \% [1]. Weak children, who have various genetic and innate features, have more chances to survive and their number increases every day.

There is a special picture for two diseases: ADHD and autism. Now both diseases are associated with different genetic causes that lead to certain psychophysiological mechanisms, but nevertheless, their diagnosis is interrelated. Moreover, very often the child is first diagnosed with ADHD, and then change it to "autism spectrum disease".

Autism spectrum disorder (ASD) comprises a continuum of heterogeneous neurodevelopmental disorders with early onset, persistent social deficits, and restricted as well as repetitive behavioral patterns. Attention-deficit and hyperactivity disorder (ADHD) is characterized by patterns of inattention, hyperactivity, and impulsivity and very often constitutes the most-frequently diagnosed co-occurring disorder in children with autism [2] and. Although according to current diagnostic practices a co-occurring presentation would permit the concurrence.

In version 4 of the Diagnostic and Statistical Manual of Mental Disorders ( $4^{\text {th }}$ ed., text Rev.; DSM-IV-TR; APA, 2000), it was not possible to combine ASD and ADHD. Now this has become possible due to numerous studies. 2,212 subjects (1,009 adults and 1,126 children) were analyzed. $770(35.3 \%)$ of the sample were women. The subjects were taken from the Netherlands Autism Register (NAR) [4]. It was shown that autism is often masked by ADHD symptoms. Since only one diagnosis is possible, autism was diagnosed on average 1.8 months later (the age is 1.5 for boys and 2.6 for girls).

The common causes of the disease include the behavior of retrotransposons. Retrotransposons (also called transposons via intermediate RNA) are genetic elements that can amplify themselves in the genome. These DNA sequences use a "copy and paste" mechanism, whereby they are first transcribed into RNA and then converted back to identical DNA sequences via reverse transcription, and these sequences are then inserted into the genome at target sites. In humans, retro elements take up $42 \%$ of the DNA.

Retroelements (RE) make up a significant part (up to $50 \%$ ) of the human genome. RE carry a large number of different regulatory elements and are able to move through the genome using retrotransposition. 
Retro elements can influence both the expression of nearby genes and the functioning of the genome as a whole.

All retro elements can be divided into 2 large groups: LTR not containing and LTR containing retrotransposons. These groups differ both in terms of structure and the mechanism of reproduction. The first group includes Autonomous LINE and non-Autonomous SINE elements.

Full-size LINE elements (Long Interspersed Nuclear Elements) have a length of 4-6 thousand p. o. and contain two open reading frames (ORF - Open Reading Frame) and a poly a sequence at the 3' end. The full-size LINE nucleotide sequence encodes the enzymes necessary for their reproduction, so this class of elements is considered Autonomous. Along the edges of the LINE elements, there is a short straight repeat of 7-20 p. o., which is a duplication of the target site.

Full-size LINE elements (Long Interspersed Nuclear Elements) have a length of 4-6 thousand p. o. and contain two open reading frames (ORF - Open Reading Frame) and a poly a sequence at the 3' end. The full-size LINE nucleotide sequence encodes the enzymes necessary for their reproduction, so this class of elements is considered Autonomous. Along the edges of the LINE elements, there is a short straight repeat of 7-20 p. o., which is a duplication of the target site.

We found that in humans, out of 516 thousand L1 sequences, only 150 are currently active (able to move independently) [3].

On average, 80 cases of L1 integration occur per neuron / Mobile elements of the genome were first described in the 40s of the last century by Barbara McClintock, who received the Nobel prize in medicine in 1983 [4].

To sum up, for the formation of an individual profile of gene expression in the neuron, in addition to the classical described mechanisms of differentiation, the most important is the phenomenon of somatic mosaicism due to the process of L1 retrotransposition. The number of such events and their localization is significant as they can contribute to the development of both autism and ADHD.

1. Nikolaeva E., Merenkova V. The Effect of a Mother's Level of Attachment and Her Emotional Intelligence on a Child's Health during Its First Year of Life // Psychology. 2013. № 5(4). P. 483-487. 
2. Belardinelli C., Raza M., Taneli T. Comorbid Behavioral Problems and Psychiatric Disorders in Autism Spectrum Disorders // J Child Dev Disord. 2016. № 2. P. 2-9.

3. Brouha B., Schustak J., Badge R. M., Lutz-Prigge S., Farley A.H., Moran J. V., Kazazian H.H. Jr. Hot L1s account for the bulk of retrotransposition in the human population // Proceedings of the National Academy of Sciences of the USA. 2003. № 1009. P. 5280-5285.

4. McClintock B. Controlling elements and the gene // Proceedings of the National Academy of Sciences of the USA. 1956. № 21. P. 197-216. 